\title{
Carrier estimations in Duchenne muscular dystrophy families in Northern Ireland using RFLP analysis
}

\author{
E D Kelly, C A Graham, A J M Hill, N C Nevin
}

\begin{abstract}
Intragenic RFLP analysis was used to provide carrier risk estimations on 100 possible female carriers from 22 Duchenne muscular dystrophy families. This enabled $78 \%$ of possible carriers to be assigned high or low risks $(>90 \%$ or $<10 \%)$ as opposed to $26 \%$ assigned low risk on pedigree data alone. When a single polymorphism is not informative the use of haplotype analysis for carrier estimations is illustrated for one family.
\end{abstract}

Duchenne muscular dystrophy (DMD) is one of the most common $\mathrm{X}$ linked recessive disorders with an incidence of 1 in 3500 male births. It has a high mutation rate and approximately one third of cases are the result of de novo mutations. ${ }^{1}$ The DMD gene has been localised to the $\mathrm{Xp} 21.2$ region by a variety of cytogenetic and molecular techniques. ${ }^{2} \mathrm{~A}$ series of intragenic probes which show several high frequency, site type, restriction fragment length polymorphisms (RFLPs) can be used for gene tracking and thus carrier estimation and prenatal diagnosis in DMD families. ${ }^{34}$

Recently the entire coding sequence of the DMD gene has been cloned and around 70 exons are spread over approximately 2 megabases of DNA. Analysis of DMD males with CDNA probes has shown that 60 to $70 \%$ have mutations causing exon deletion. ${ }^{5}$

Linkage studies with the intragenic pERT and XJ probes have shown an average recombination rate of around $5 \%,{ }^{46}$ probably owing to the large size of the gene and the heterogeneity of the mutation site. In this study a combination of seven polymorphisms have been used for gene tracking in $22 \mathrm{DMD}$ families and revised risks have been computed for 100 females who are possible carriers of the DMD gene. Department of Medical Genetics, The Queen's Univer-
sity of Belfast, Tower Block Floor A, Belfast City
Hospital, Belfast BT9 7AB.

E D Kelly, C A Graham, A J M Hill, N C Nevin

Correspondence to Dr Graham.

Received for publication 13 June 1989.

Revised version accepted for publication 1 September 1989.
Materials and methods

SAMPLE COLLECTION AND DNA EXTRACTION

Venous blood $(20 \mathrm{ml})$ was collected into $50 \mathrm{ml}$ centrifuge tubes containing $5 \mathrm{ml}$ of $5 \%$ EDTA, pH 8.0 , as anticoagulant. The red cells were lysed and the white cell pellet collected by centrifugation. This could be stored for up to one month at $-20^{\circ} \mathrm{C}$ before processing. DNA extraction was carried out by a method based on that of Jeanpierre. ${ }^{7}$

DNA PROBES

Four intragenic DNA probes were used to detect seven different polymorphisms (fig 1). A recombination rate of $5 \%$ was used in all risk calculations based on these probes.

\section{DNA ANALYSIS}

DNA (5 to $10 \mu \mathrm{g}$ ) from the appropriate family members was digested with restriction enzymes according to the manufacturers' instructions. The resulting fragments were size fractionated by electrophoresis in $0.8 \%$ agarose gels (Bethesda Research Laboratories). The fragments were transferred to nylon membranes (Hybond- $\mathrm{N}$, Amersham) by the method of Southern ${ }^{8}$ or by vacuum blotting using the Hybaid Vacuaid system. The DNA was covalently bound to the membrane by UV photocrosslinking (305 $\mathrm{nm}$ for 90 seconds). The probe insert was electrophoretically purified and $50 \mathrm{ng}$ was labelled with a ${ }^{32} \mathrm{P}$-dCTP using the random hexanucleotide primer method of Feinburg and Vogelstein ${ }^{9}$ (Multiprime Kit, Amersham). Unincorporated nucleotides were removed by gel filtration on a Sephadex G-50 column and the specific activity of the probe was measured by scintillation counting (usually 5 to $10 \times 10^{8} \mathrm{dpm} / \mu \mathrm{g}$ DNA). The membrane was incubated for a minimum of four hours at $42^{\circ} \mathrm{C}$ in a formamide based prehybridisation buffer ( $50 \%$ formamide, $0.1 \%$ Denhardt's, $1 \%$ SDS, $5 \times$ SSPE, $5 \%$ dextran sulphate, and $400 \mu \mathrm{g} / \mathrm{nl}$ sheared salmon sperm DNA). The hybridisation solution was the same without salmon sperm DNA, which was added to the labelled probe before denaturation. The membrane was hybridised at $42^{\circ} \mathrm{C}$ overnight and then washed in $2 \times$ SSPE at room temperature followed by $65^{\circ} \mathrm{C}$ washes at in- 


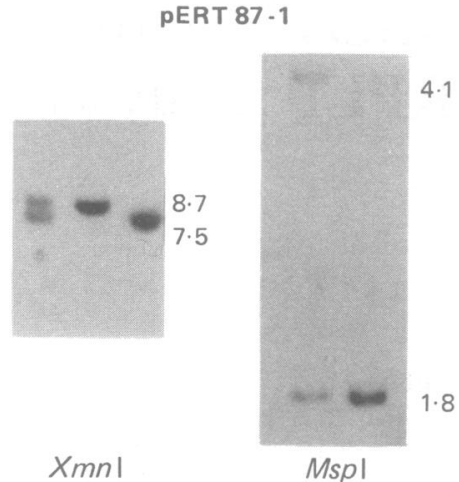

PERT $87-15$

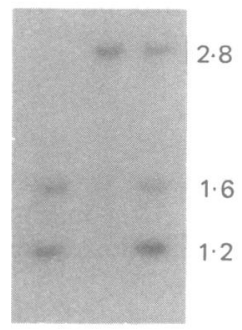

$X m n l$

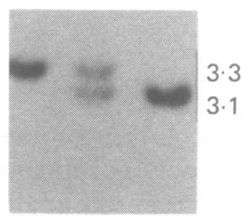

Taql
PERT $87-8$

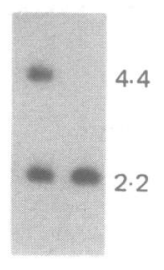

Bst XI

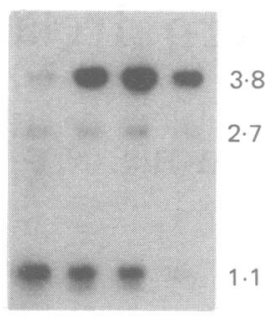

Taql

XJ 1.1

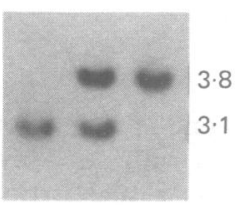

Taql

Figure 1 Autoradiographs of ${ }^{32} P-d C T P$ labelled probes hybridises to restriction digested total human pERT and Xf intragenic DMD probes are shown with allele sizes in kilobases. creasing stringency as required. The membranes were then placed in $x$ ray cassettes with intensifying screens and Fuji RX medical $x$ ray film and exposed at $-70^{\circ} \mathrm{C}$ for one to four days before development.

\section{Results and discussion}

RFLP analysis has been carried out on a total of 22 DMD families in Northern Ireland. Twelve of the families had isolated cases of DMD and the carrier status of the mother was unknown. In a further two families the mother was judged to be a carrier since she had more than one affected son. The remaining families showed a definite familial mode of inheritance.

Definite carriers and mothers of affected males were screened for heterozygosity with a series of six RFLPs using the intragenic pERT87 and XJ probes; examples of each polymorphism are shown in fig 1 . The $M s p I / 87-1$ polymorphism was used to provide information in only one family but was not included in routine screening. Thirty four out of 39 females $(87 \%)$ tested have proved informative with at least one probe. $X m n I$ and $T a q I$ digests were run initially since these enzymes detect five of the six RFLPs. The $X m n \mathrm{I} / 87-1$ and TaqI/87-15 XJ polymorphisms have proved the most informative and simple to use.
Complete RFLP analysis has been carried out on 20 families using a combination of the markers describech above. Generally, once informativeness was established for a family carrier estimations were calculated on the $\vec{E}$ basis of RFLP data from one, or at the most two $\$$ probes. The final risk estimation on a female was calculated from a combination of pedigree and RFLP information using the MLINK computer program CK values from females have not been included in the overall risk estimation since a standard range of values for obligate carriers had not been established; a high CK value is, however, considered when counsellingo females at risk. This is particularly important in simplex families where RFLP analysis may give little information. In familial cases of DMD the majority of female relatives can be assigned $5 \%$ or $95 \%$ carrie? risks based on RFLP analysis. High carrier risks can only be obtained when the disease is familial, and accurate prenatal diagnosis can be offered to suct females.

The accuracy with which analysis can be carried out in isolated cases is much more variable. When the carrier status of a mother with an affected boy is్ unknown it is not possible to raise the risk of any of her daughters above the mother's risk, though theiro risks could be reduced if they had inherited the alternative maternal allele to the affected boy. In three 
of the sporadic families the risk to the second degree female relatives was reduced to a negligible value since the disease associated allele was derived from the grandfather of the affected male. However, the mother's risk and that of any daughters could not be altered in this way. Six female sibs of affected males from four families were virtually excluded as carriers on the basis of not having inherited the disease associated allele from their mother. In a further four families the risks to possible carriers was significantly reduced since it was shown that both affected and normal males in the same family shared a common allele. In isolated cases this is more likely to be the result of a new mutation in the affected male than recombination.

Risks based on RFLP analysis have now been obtained on 100 females from the 22 families analysed. Each subject's risk before and after RFLP analysis was calculated using the MLINK computer program. The results are summarised in fig 2. Before RFLP analysis only $26 \%$ of females had a carrier risk of $10 \%$ or less, with the remainder showing an even spread in risks up to $60 \%$. However, on the basis of RFLP analysis $67 \%$ of females have risks of $10 \%$ or less. A further $11 \%$ of the females now have a high risk of greater than $90 \%$ and are solely from families where DMD shows familial inheritance. The risks for the remaining $23 \%$ of females are fairly evenly spread. In $78 \%$ of the females the risks were altered through direct RFLP results. However, for the remaining $22 \%$ RFLP data were either not available because no DNA was obtained or proved unnecessary. The risks for these females were altered on the basis of RFLP data available for close relatives.

Many DMD families prove unsuitable for RFLP analysis with a single polymorphism because the DNA from important family members is not available. We provide an example of such a DMD family in which the proband was concerned about her carrier status and was pregnant at the time. The only living affected male was a cousin whose mother was dead, as were the maternal grandparents, making routine RFLP analysis impossible. Deletion screening of the affected male proved negative and therefore we attempted a carrier estimation on the proband on the basis of haplotype analysis. RFLP data was obtained on four polymorphisms as shown in fig 3. The affected male (III.3) and his normal uncle (II.1) both share the same haplotype, indicating that the grandmother (I.1) was homozygous for all the alleles. The haplotype of her daughter (II.2), a definite carrier, allowed the haplotype of the grandfather (I.2) to be inferred. The proband (III.2) has inherited the normal maternal chromosome, as has her half sister (III.1). The two polymorphisms of the pERT87-15 probe were sufficient to define an informative haplotype and were used for risk calculation using MLINK. Their carrier risks have therefore been reduced from $50 \%$ to $8.8 \%$ and the proband now has a 1 in 45 chance of having an affected boy. The use of haplotypes can have an important, if limited, application in such families but is no more accurate for use on a routine basis.

\section{Conclusion}

We have provided carrier estimations on 100 females from 22 DMD families using RFLP analysis. Key females were screened for heterozygosity with the probes previously described and this strategy has

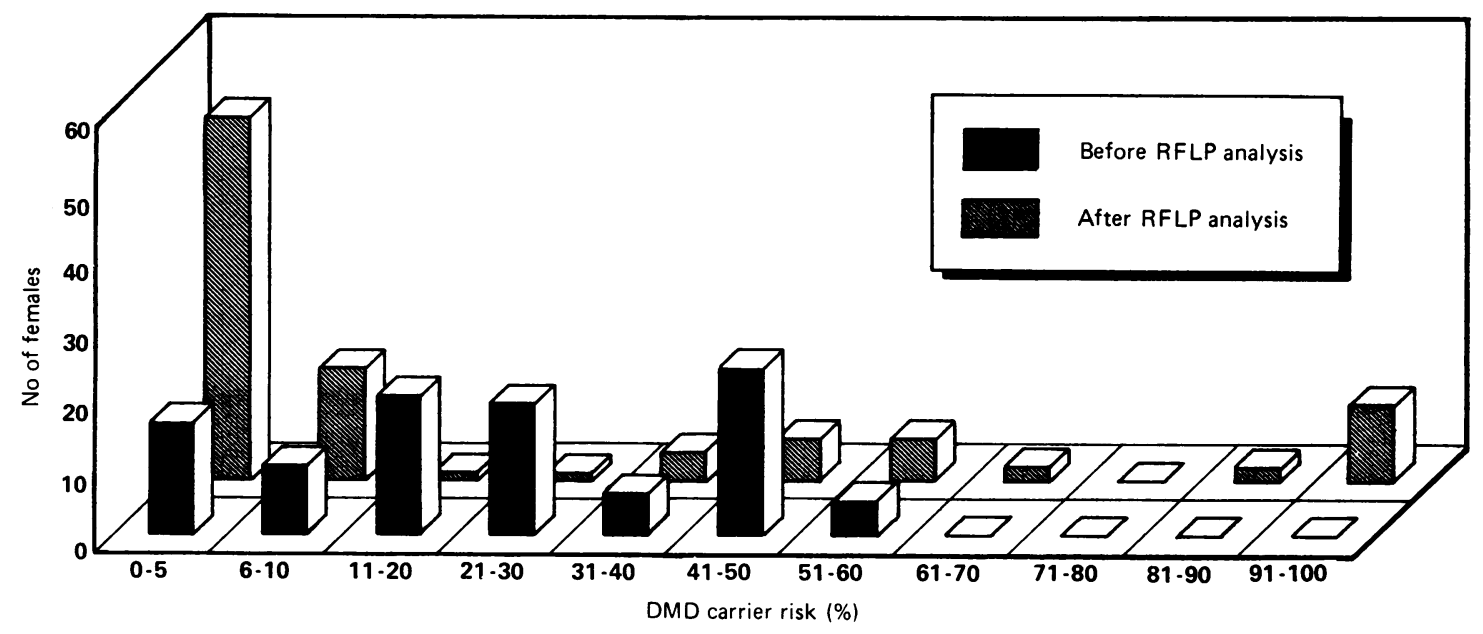

Figure 2 Diagram illustrating the effectiveness of RFLP analysis in providing carrier risk estimations in DMD. All risks were calculated using MLINK from LINKAGE v3.5 and risks before RFLP analysis were based on pedigree data only. 


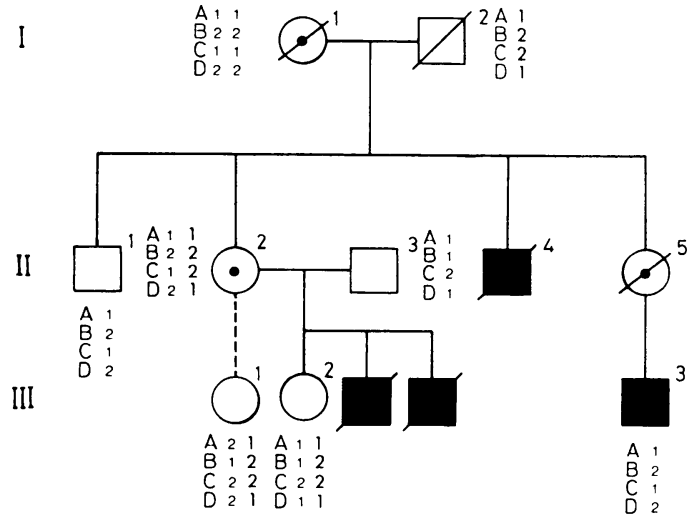

Figure 3 Pedigree illustrating the use of haplotype analysis for carrier estimations. The haplotypes of the affected boy III.3 and his normal uncle II.2 are the same, implying that the grandmother I.1 was homozygous for all probes. However, from the haplotypes of obligate carrier II.2 the grandfather's haplotype could be inferred, and both at risk females III.I and III. 2 were shown to have inherited the grandpaternal haplotype from their mother, thus reducing their carrier risks from $50 \%$ to $8.8 \%$ (MLINK). A: pERT87.1/XmnI, B: pERT87.15/XmnI, C: pERT87.15/TaqI, D:XF1.1/TaqI.

proved both effective and efficient for future analysis of the families. Markers flanking the DMD gene were not used in this study because of the difficulty in obtaining two informative bridging probes. However, this strategy can improve the accuracy of risk estimations, that is, informative bridging probes showing $10 \%$ recombination each can give $99 \%$ risk estimates.

Our results reflect the advantages and limitations of the use of intragenic RFLP analysis for providing carrier estimations on females at risk. Such analysis is both useful and accurate where the disease is obviously familial. Risks can be calculated with a maximum of $95 \%$ certainty and high risk females then have the option of an accurate prenatal test.

However, only eight of our families show classical familial inheritance with most of the remainder being isolated cases. The usefulness of RFLP analysis in such families is much more variable. We were able to reduce the risks of $56 \%(20 / 36)$ of females, with prior risks of 10 to $60 \%$, to below $6 \%$, thus removing the need for non-deletion based prenatal tests in these females.

Overall there has been a polarisation of risks at the two extremes after RFLP analysis with $78 \%$ of females now within either high or low risk categories. However, for approximately $20 \%$ of females RFLP analysis has been of little benefit. These females have generally inherited the disease associated allele carried

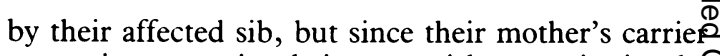
status is uncertain their own risks remain in the middle range. The only option for such females, if deletion screening is not possible, is exclusion analysis: on male fetuses to confirm the presence or absence of the disease associated allele, though the high number of false positives may be unacceptable to many women.

In the future the application of RFLP analysis max become more limited as effective counselling reduces the number of female carriers requiring analysis and sporadic cases contribute increasingly to the per $\overrightarrow{0}$ centage of families seeking analysis. Thus, alternative methods of carrier detection will be required. In casesw where the affected male shows an exon deletion then exon dosage assessment may be appropriate if methods can be accurately standardised. Junctioni fragments are also useful although these are onlyh detected in a small number of cases. Perhaps the mos: widely applicable methods for the detection of deletioncarriers will be pulsed field gel electrophoresis ${ }^{10}$ and cosmid hybridisation. ${ }^{11}$ The use of antidystrophinantibodies may be appropriate in non-deletion families where females show equivocal serum $\mathrm{CK}$ values.

We wish to thank Dr L M Kunkel for providing the pERT probes and Dr R G Worton for the XJ1.leo probe. This work was supported by a Research a 808 Development grant from the Department of Hea and Social Services (Northern Ireland).

1 Emery AEH. In: Emery AEH, Rimoin DL. Principles and practice of medical genetics. Edinburgh: Churchill Livingstone, 1983.

2 Worton RG, Burghes AHM. Molecular genetics of Duchenne and Becker muscular dystrophy. Int Rev Neurol 1988;29:1-75.

3 Old JM, Davies KE. Prenatal diagnosis of Duchenne muscularo dystrophy by DNA analysis. $\mathcal{F}$ Med Genet 1986;23:556-9.

4 Lindlof $M$, Sistonen $P$, de la Chappelle A. Linked polymorphic DNA markers in the prediction of X-linked muscular dyseق trophy. Ann Hum Genet 1987;51:317-28.

5 Read AP, Mountford RC, Forrest SM, Kenrick SJ, Davies KE, 긍 Harris R. Patterns of exon deletion in Duchenne and Becker? muscular dystrophy. Hum Genet 1988;80:152:6.

6 Donald JA, Morgan G, Chen JD, Serravalle S, Colley P, Denton MJ. Recombination between Duchenne muscular dystrophy and DNA marker DXS164 (pERT 87). Lancet 1987;i:39-40.

7 Jeanpierre M. A rapid method for the purification of DNA from blood. Nucleic Acids Res 1987;15:9611.

8 Southern EM. Detection of specific sequences among DNA음 fragments separated by gel electrophoresis. F Mol Biol 1975;98: 503-7.

9 Feinburg AP, Vogelstein B. A technique for radiolabelling DNA fragments to high specific activity. Anal Biochem 1984;137: N 266-7.

10 Chen J, Denton MJ, Morgan G, Pearn JH, Mackinlay AG. The use of field inversion gel electrophoresis for deletion detection in Duchenne muscular dystrophy. Am f Hum Genet 1988;42. 777-80.

11 Blonden LAJ, den Dunnen JT, van Paassen HMB, et al. High resolution deletion breakpoint mapping in the DMD gene by
whole cosmid hybridisation. Nucleic Acids Res 1989;17:5611-28. 